\title{
Noncoding RNAs of Plant Viruses and Viroids: Sponges of Host Translation and RNA Interference Machinery
}

\author{
W. Allen Miller, ${ }^{1,2}$ Ruizhong Shen, ${ }^{1,2}$ William Staplin, ${ }^{2}$ and Pulkit Kanodia ${ }^{1,2}$ \\ ${ }^{1}$ Interdepartmental Genetics \& Genomics Program and ${ }^{2}$ Department of Plant Pathology \& Microbiology, lowa State University, \\ Ames, IA 50011, U.S.A.
}

Submitted 7 October 2015. Accepted 6 January 2016.

\begin{abstract}
Noncoding sequences in plant viral genomes are well-known to control viral replication and gene expression in cis. However, plant viral and viroid noncoding (nc)RNA sequences can also regulate gene expression acting in trans, often acting like 'sponges' that bind and sequester host cellular machinery to favor viral infection. Noncoding sequences of small subgenomic (sg)RNAs of Barley yellow dwarf virus (BYDV) and Red clover necrotic mosaic virus (RCNMV) contain a cap-independent translation element that binds translation initiation factor eIF4G. We provide new evidence that a sgRNA of BYDV can globally attenuate host translation, probably by sponging eIF4G. Subgenomic ncRNA of RCNMV is generated via $5^{\prime}$ to $3^{\prime}$ degradation by a host exonuclease. The similar noncoding subgenomic flavivirus (sf)RNA, inhibits the innate immune response, enhancing viral pathogenesis. Cauliflower mosaic virus transcribes massive amounts of a 600-nt ncRNA, which is processed into small RNAs that overwhelm the host's RNA interference (RNAi) system. Viroids use the host RNAi machinery to generate viroid-derived ncRNAs that inhibit expression of host defense genes by mimicking a microRNA. More examples of plant viral and viroid ncRNAs are likely to be discovered, revealing fascinating new weaponry in the host-virus arms race.
\end{abstract}

Plant viruses have small and compact genomes. Thus, noncoding regions are limited to a very small portion of a typical plant virus genome. In fact, sequence space is at such a premium that many plant viral genomes encode overlapping genes (Fig. 1). Because so little sequence is noncoding, few noncoding (nc) RNAs are known in plant viruses. Viroids also generate ncRNAs, which are discussed here. Noncoding satellite RNAs are discussed elsewhere in this Molecular Plant-Microbe Interactions Focus issue (Palukaitis 2016) and by Shimura and Masuta (2016). In contrast to plant viral RNAs, ncRNAs that regulate host and viral gene expression are abundant in herpes viruses of animals. Their large (>100 kbp) DNA genomes encode numerous micro (mi)RNAs and other ncRNAs that manipulate expression of host and viral genes (Guo and Steitz 2014). For reasons that are beyond the scope of this article, no such large viruses exist in plants (Dolja and Koonin 2011). The major known roles of noncoding regions in plant viral RNA are to control RNA synthesis, encapsidation, or translation in cis, as part of the viral genomic RNA or viral

Current address for R. Shen: Department of Medicine-Division of Gastroenterology and Hepatology, University of Alabama at Birmingham, Birmingham, AL 35294, U.S.A.

Corresponding author: W. A. Miller; E-mail: wamiller@iastate.edu

@ 2016 The American Phytopathological Society messenger (m)RNAs (Newburn and White 2015). Some examples of plant viral RNAs that act in trans via their noncoding regions are known in the luteoviruses, tombusvirids, and pararetroviruses and are the subject of this review, as are viroids, which, with one exception, are entirely noncoding (See also Gago-Zachert 2016). We also provide new data supporting the role of plant viral ncRNAs as inhibitors of host translation. It is likely that many more plant viral ncRNAs remain to be discovered.

Barley yellow dwarf virus and other luteoviruses.

Viruses in genus Luteovirus of the Luteoviridae family and viruses in the related Tombusviridae family produce $3^{\prime}$ coterminal subgenomic (sg)RNAs (Domier 2012; Jiwan and White 2011; Miller and Koev 2000; Rochon et al. 2012). Most of these sgRNAs serve as mRNAs to allow translation of $5^{\prime}$-distal genes in the genomic RNA. However, others correspond only to the $3^{\prime}$ untranslated region (UTR) and are, thus, noncoding (nc)sgRNAs. Also, noncoding regions of some of these sgRNAs that do contain coding regions, i.e., open reading frames (ORFs), may regulate host or viral RNA gene expression, or both, in trans, in the manner of a ncRNA.

In the infected cell, luteoviruses generate two or three sgRNAs, depending on the virus (Fig. 1). All share the $3^{\prime}$ terminus of genomic RNA (Kelly et al. 1994; Yamagishi et al. 2003). sgRNA1 of Barley yellow dwarf virus (BYDV) and other luteoviruses consists of the $3^{\prime}$ half of the viral genome and serves as mRNA for four ORFs, translated by noncanonical means, that code for coat protein $(\mathrm{CP})$ and other proteins involved in virus movement in the plant or its aphid vector (Brown et al. 1996; Dinesh-Kumar and Miller 1993; Smirnova et al. 2015). Thus, sgRNA1 is a supercoding mRNA. On the other hand, 800-nt sgRNA2 of BYDV encodes only the small ORF 6, the product of which varies in size from 4.3 to $7.2 \mathrm{kDa}$, depending on the virus isolate (Chalhoub et al. 1994). This protein (P6) has not been detected in infected cells (Shen et al. 2006). A construct designed to express P6 was shown to suppress RNA silencing (Liu et al. 2012), but the authors did not detect the P6 protein, so they did not rule out the possibility that the RNA encoding P6, which includes the $5^{\prime}$ end of sgRNA2, is the actual silencing suppressor rather than the predicted protein. sgRNA3 encodes no ORFs and consists of the 3 '-terminal $330 \mathrm{nt}$ of the BYDV genome (Kelly et al. 1994). sgRNA2 and sgRNA3 are present in many tens-fold molar excess over sgRNA1 and genomic RNA (Kelly et al. 1994; Koev and Miller 2000).

RNAs of luteoviruses and the tombusvirids lack a 5' cap and a poly(A) tail. Instead, for protein synthesis, they rely on a capindependent translation element (CITE) located near the $5^{\prime}$ end of the $3^{\prime}$ UTR of the genomic RNA (Simon and Miller 2013). The 3' CITE of BYDV, called the BYDV-like translation 
element (BTE), is located between ORF 5 and ORF 6, about $800 \mathrm{nt}$ from the $3^{\prime}$ end of the genome (Fig. 1). The BTE powerfully stimulates translation of the viral genomic RNA and sgRNA1 (Fan et al. 2012; Rakotondrafara et al. 2006; Wang et al. 1997) by binding with high affinity to eIF4G, which is the scaffolding subunit of the key translation initiation heterodimer eIF4F (Kraft et al. 2013; Treder et al. 2008). The BTE is in the 5' UTR of sgRNA2 (Fig. 1). sgRNA2 regulates translation of viral genomic RNA and sgRNA1 in trans, via its BTE (Shen et al. 2006; Wang et al. 1999). Because of the BTE at its 5 ' end, which binds eIF4G, sgRNA2 strongly trans-inhibits translation of genomic RNA but only slightly inhibits translation of sgRNA1 (Wang et al. 1999). In infected cells, this interaction may serve as a switch to favor translation of late genes (virus movement and packaging) from sgRNA1 over translation of early (RNA synthesis) genes from the genomic RNA (Shen and Miller 2004; Shen et al. 2006). This differential effect on translation of BYDV genomic RNA and sgRNA1 by sgRNA2 is conferred by their different 5' UTRs. Genomic RNA has a highly structured 5' UTR (Guo et al. 2001), whereas sgRNA1 has relatively unstructured and, thus, less eIF4F-dependent 5' UTR (Shen et al. 2006). To summarize, the noncoding portion of sgRNA2 regulates translation of other viral genes, and it acts like a regulatory ncRNA (even though it contains a small ORF).

Owing to its high abundance and high affinity for eIF4G, we expect sgRNA2 to attenuate translation of host mRNAs as well as the other viral RNAs. Indeed, we showed that sgRNA2 inhibits translation (in trans) of a capped, polyadenylated luciferase
mRNA lacking any viral sequence (Shen and Miller 2004). To determine if translation inhibition occurs in infected cells, this reporter mRNA was electroporated into oat protoplasts $24 \mathrm{~h}$ after infection with wild-type BYDV-PAV (PAV6) or mutant BYDVPAV with a point mutation preventing synthesis of sgRNA2 (PAV6 $\Delta$ SG2) (Koev and Miller, 2000), and luciferase activity was measured after $4 \mathrm{~h}$. Both sets of cells accumulated similar amounts of viral genomic RNA and sgRNA 1 (Fig. 2A, right panel). Importantly, luciferase expression averaged one-half as much in the PAV6-infected cells compared with the PAV6 $\Delta$ SG2-infected cells (Fig. 2A, left panel). Thus, sgRNA2 accumulation inhibits translation of nonviral mRNA, leading us to predict that host mRNAs would be similarly inhibited.

To detect global reduction of host translation, we transgenically expressed sgRNA2 via an estradiol-inducible promoter in Arabidopsis. Arabidopsis Col-0 plants were transformed with either empty vector, vector expressing full-length sgRNA2, or fulllength sgRNA2 containing a four-base duplication in the natural BamHI site that completely inactivates the the BTE (sgRNA2BF), driven by an estradiol-inducible promoter. Binary vectors pERSG2 and pERSG2BF were constructed by inserting polymerase chain reaction-amplified BYDV sgRNA2 and sgRNA2BF into XhoI/SpeI-cut pER8 (Zuo et al. 2000), respectively. Transformation of Agrobacterium tumefaciens GV3101::pMP90 was done as by Shen and Forde (1989), using a MicroPulser (BioRad). Transformation of Arabidopsis thaliana Col-0 ecotype was carried out by floral dip as reported by Clough and Bent (1998). T3 or T4 seeds were used for experiments. Polysome profiles
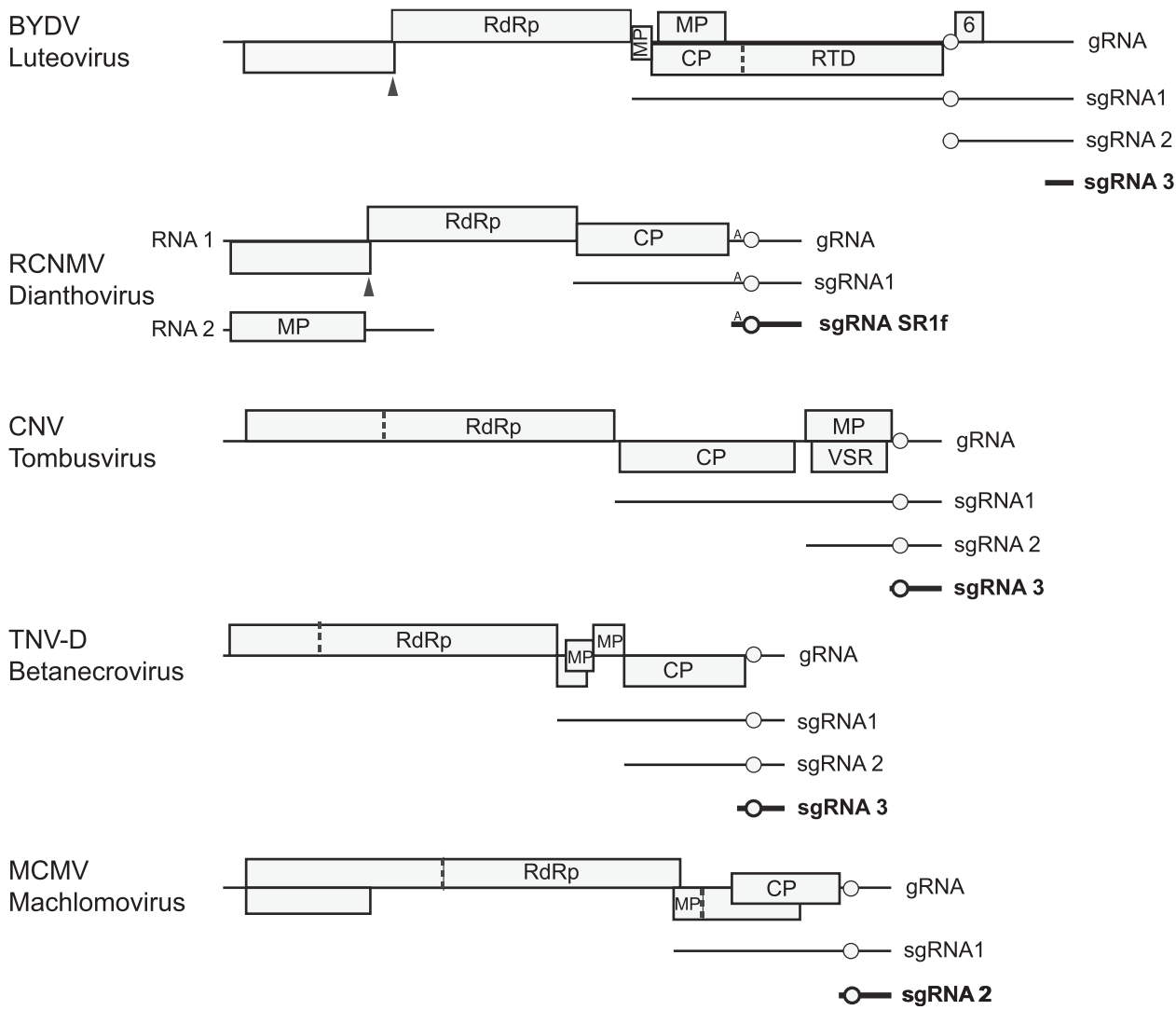

Fig. 1. Genome organizations of RNA viruses known to produce noncoding subgenomic (sg)RNAs. Noncoding sgRNAs are indicated in bold. Luteoviruses Soybean dwarf virus and Bean leafroll virus lack open reading frame (ORF) 6 and sgRNA2, while luteovirus Rose spring dwarf-associated luteovirus has two additional small ORFs downstream of ORF 6. The 5' ends of Cucumber necrosis virus (Johnston and Rochon 1995) and Tobacco necrosis virus-D (Jiwan et al 2011) noncoding sgRNAs have not been mapped precisely. Circles indicate the position of the $3^{\prime}$ cap-independent translation element (CITE). A small A on Red clover necrotic mosaic virus (RCNMV) RNAs indicates the A-rich sequence needed for maximal CITE activity. Solid triangles indicate ribosomal frameshift sites and dashed lines indicate leaky stop codons. BYDV = Barley yellow dwarf virus, $\mathrm{RdRp}=\mathrm{RNA}$-dependent RNA polymerase, MP $=$ movement protein, $\mathrm{CP}=$ coat protein, $\mathrm{RTD}=$ coat protein readthrough domain, VSR = viral suppressor of RNA interference. 
were obtained from seedlings treated with dimethyl sulfoxide (DMSO) alone (mock-induced) or with $\beta$-17-estradiol (10 mM) in DMSO (induced) by grinding 0.7 to $2 \mathrm{~g}$ of plant material under liquid nitrogen. Extraction buffer $(200 \mathrm{mM}$ Tris- $\mathrm{HCl}, \mathrm{pH}$
8.5, $200 \mathrm{mM} \mathrm{KCl}, 30 \mathrm{mM} \mathrm{MgCl}$, $10 \mathrm{mM}$ EGTA, $200 \mathrm{mM}$ sucrose, $10 \mathrm{mM} \beta$-mercaptoethanol, $2.5 \mathrm{mM}$ DTT, $0.5 \mathrm{mg}$ of heparin per milliliter, $5 \mu \mathrm{g}$ of proteinase $\mathrm{K}$ per milliliter, $100 \mu \mathrm{g}$ of chloramphenicol per milliliter, $50 \mu \mathrm{g}$ of cyclohexamide per
A

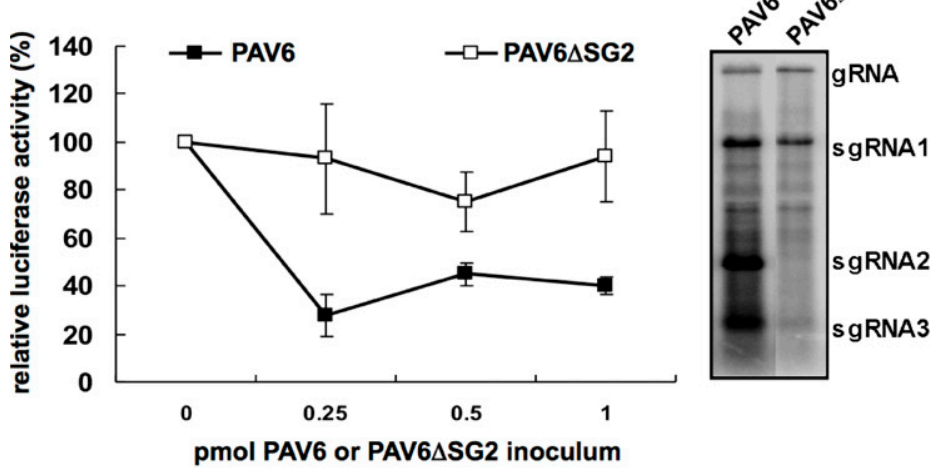

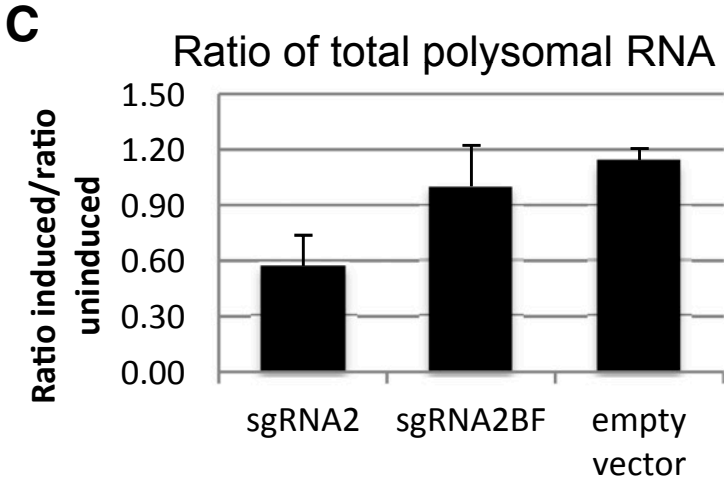

mock-induced

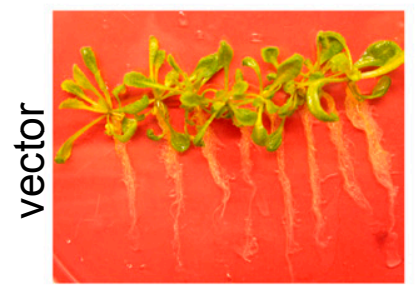

induced

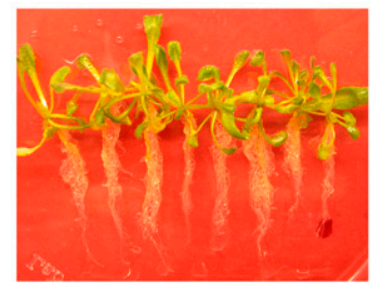

405605805 Polysomes
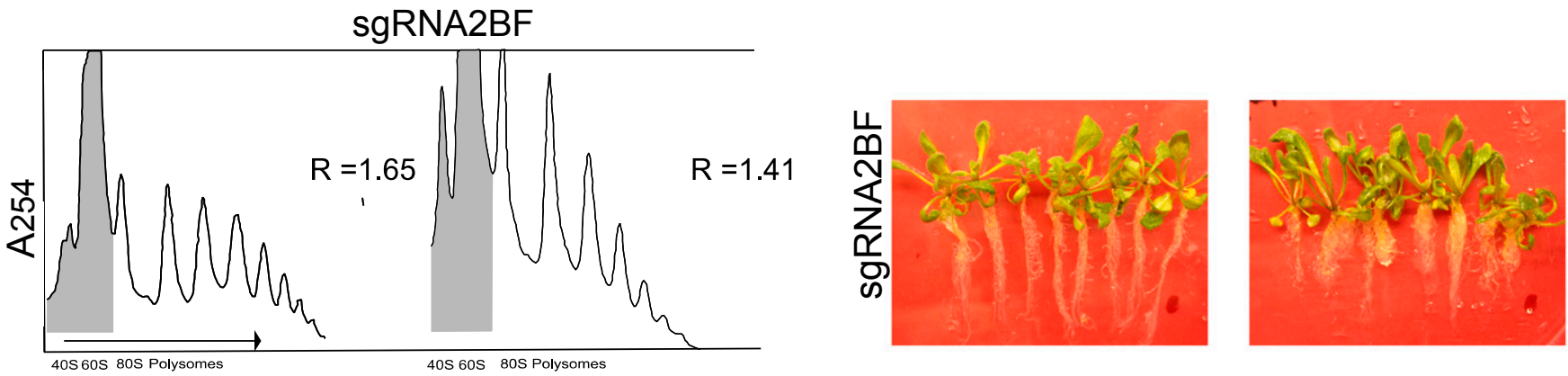

sgRNA2
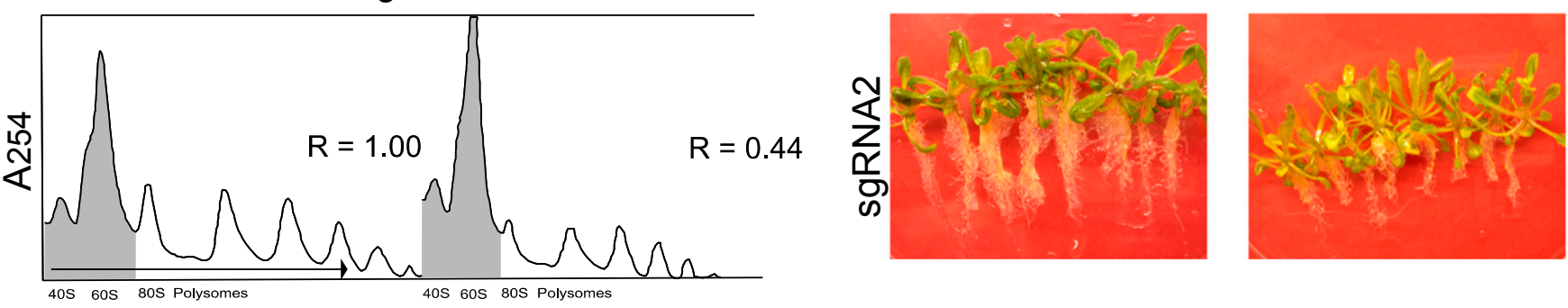

Fig. 2. Effect of expression of Barley yellow dwarf virus (BYDV) subgenomic (sg)RNA2 on translation and plant phenotype. A, The effect of sgRNA2 on translation of capped, polyadenylated, nonviral luciferase mRNA in infected oat protoplasts. Oat protoplasts infected with the indicated transcript were electroporated with firefly luciferase mRNA, and then, assayed for luciferase activity after $4 \mathrm{~h}$. B, Polysome profiles of plants expressing sgRNA2. The polysome profiles are representative of profiles collected in duplicate (pER8 vector and sgRNA2BF) or quadruplicate (sgRNA2). To calculate the ratio (R) of translating to nontranslating RNA, the combined areas of the monosome (80S) and polysome peaks (unshaded areas) were divided by the areas of the 40S plus $60 \mathrm{~S}$ peaks (shaded). ( $\mathrm{R}$ indicated beside polysome profiles.) $\mathrm{C}$, The changes due to expression of the indicated RNA are plotted showing averages (with standard error) from four separate experiments, such as the one shown in B. D, Transgenic Arabidopsis seedlings after 12 days of growth on media containing estradiol (induced) or DMSO buffer only (mock-induced). 
milliliter) was added to the pulverized tissue at a ratio of $0.5 \mathrm{ml}$ to $1 \mathrm{~g}$ of plant material (Hollingsworth et al. 1998). Upon thawing, the homogenates were centrifuged at $16,000 \times g$ for $10 \mathrm{~min}$, supernatants were layered on a sucrose cushion $(1.75 \mathrm{M}$ sucrose, $40 \mathrm{mM}$ Tris- $\mathrm{HCl}$, pH 9, $30 \mathrm{mM} \mathrm{MgCl} 2,200 \mathrm{mM} \mathrm{KCl}$, $5 \mathrm{mM}$ EGTA, $10 \mathrm{mM} \beta$-mercaptoethanol) and were centrifuged in a SW 50.1 rotor at $234,000 \times g$ for $18 \mathrm{~h}$. Pelleted polysomes were washed and resuspended in $50 \mu \mathrm{l}$ of resuspension buffer (200 mM Tris-HCl, pH 8.5, 60 mM KCl, 30 mM MgCl $2,100 \mu \mathrm{g}$ of chloramphenicol per milliliter, $50 \mu \mathrm{g}$ of cyclohexamide per milliliter, $10 \mathrm{mM} \beta$-mercaptoethanol), and then, were layered on a 20 to $60 \%$ sucrose gradient $(40 \mathrm{mM}$ Tris- $\mathrm{HCl}, \mathrm{pH} 8.5$, $30 \mathrm{mM} \mathrm{KCl}, 10 \mathrm{mM} \mathrm{MgCl}_{2}, 10 \mathrm{mM} \beta$-mercaptoethanol), through which they were centrifuged in a SW41 rotor at 200,000 $\times g$ for $2.5 \mathrm{~h}$. Gradients were fractionated with a Brandel pump and an Isco apparatus (UA-6) absorbance monitor with a 254-nm UV filter.

High levels of sgRNA2 were detected after induction with estradiol, and total translating RNA (monosomes and polysomes relative to $40 \mathrm{~S}$ and $60 \mathrm{~S}$ ribosomal subunits) was reduced by about $50 \%$, indicating a reduction in translation of host mRNAs (Fig. 2B and C). Plants expressing empty vector and negative control plants expressing sgRNA2BF showed little change in polysomal mRNAs (Fig. 2B and C). Such a global reduction in translation caused by sgRNA2 would be expected to have an adverse effect on plant health. Indeed, 12 days after induction of sgRNA2, seedlings showed a phenotype of decreased root length and, ultimately, appeared stunted (Fig. 2D). The plants expressing sgRNA2BF showed only slight, if any, decrease in root length, and those expressing empty vector RNA were unchanged in phenotype (Fig. 2D).

The biological role of inhibition of host translation by sgRNA2 is uncertain. It may inhibit translation of host defense genes. sgRNA2 and sgRNA3 are not essential for RNA replication in protoplasts. Mutations in the promoters that prevent synthesis of these sgRNAs did not greatly reduce viral RNA accumulation (Koev and Miller 2000; Shen and Miller 2004). Surprisingly, virus containing these mutations was able to infect oat plants and accumulate to levels similar to those of wildtype virus (Miller et al. 2015). However, after passaging the sgRNA double-knockout mutants, a new sgRNA that is slightly larger than sgRNA3 appeared in some infected plants. Thus, natural selection seems to favor the presence of a small ncsgRNA similar to sgRNA3, but BYDV can replicate in the host (at least in highly susceptible oats cv. Clintland 64) under the controlled conditions of our laboratory, in the absence of both sgRNA2 and sgRNA3. We speculate that the wild-type virus would be more successful in direct competition with the mutant virus or, in different hosts or field conditions, sgRNA2 and sgRNA3 may provide a distinct advantage. In support of their importance, these sgRNAs were found in all 22 field isolates tested by Kelly et al. (1994).

Another luteovirus, Soybean dwarf virus (SbDV) generates a highly abundant 320-nt ncsgRNA similar to sgRNA3 of BYDV (Yamagishi et al. 2003). This virus appears not to produce a homolog of BYDV sgRNA2, although a faint band of about this size is visible on Northern blot hybridizations of some isolates (Yamagishi et al. 2003). Also, for the closely related Bean leafroll virus (BLRV), only sgRNA3-sized (319 nt) noncoding sgRNA was detected, although this virus contained a potential promoter at the expected site for sgRNA2 synthesis (Domier et al. 2002). Interestingly, neither SbDV nor BLRV encode a homolog of ORF 6 of BYDV (Domier et al. 2002). Yet these viruses also harbor a BTE at the 5' end of a long (700 nt) 3' UTR. In contrast, like BYDV, Rose spring dwarf-associated luteovirus (RSDaV) generates an approximately 850-nt sgRNA2 that encodes an ORF 6. Unlike other luteoviruses, RSDaV
sgRNA2 also contains two small ORFs downstream of ORF 6 (Salem et al., 2008). Whether RSDaV generates sgRNA3 is unknown. Thus, the regions of the luteovirus genome downstream of ORF 5 remain somewhat of a mystery. Depending on the virus, it can encode between zero and three ORFs, and it may or may not generate the approximately 850-nt sgRNA2. In all luteoviruses, the region downstream of ORF 5 contains the BTE and a 25-nt stem-loop downstream required for -1 frameshifting (Barry and Miller 2002) at the $5^{\prime}$ end and a structure required for RNA replication at the $3^{\prime}$ end (Koev et al. 2002). The intervening 300 to 400 bases perform no known function in trans or in cis.

\section{Red clover necrotic mosaic virus and other Tombusviridae.}

One of the few other plant viruses for which trans-acting ncsgRNAs have been identified is Red clover necrotic mosaic virus (RCNMV) (genus Dianthovirus, family Tombusviridae). Although in a different family, it is closely related to the luteoviruses (Miller et al. 2002). Unlike the luteoviruses and other tombusvirids, RCNMV has a bipartite genome consisting of genomic RNA1 and RNA2 (Okuno and Hiruki 2013). Like other tombusvirids and luteoviruses, both of these positive-sense RNAs have neither a 5' cap (Mizumoto et al. 2003) nor a poly(A) tail (Lommel et al. 1988; Xiong et al. 1989). RNA1 encodes a 27-kDa protein, p27, and an 88-kDa protein, p88, which contains an RNAdependent RNA polymerase (RdRp) motif (Fig. 1) (Lommel et al. 1988; Xiong et al. 1989). As in the luteovirus genome, the RdRp is translated via -1 ribosomal frameshifting directed by an RNA structure that requires base-pairing of a stem-loop in the $3^{\prime}$ UTR with a bulged stem-loop structure adjacent to the frameshift site (Tajima et al. 2011). A 3'-coterminal sgRNA, CPsgRNA, is generated from RNA1 and serves as mRNA for the $37-\mathrm{kDa}$ CP (Zavriev et al. 1996). RNA2 encodes a 35-kDa movement protein (MP) (Fig. 1) (Xiong et al. 1993).

RCNMV RNA1 contains a BTE in its 3' UTR to facilitate cap-independent translation (Mizumoto et al. 2003). This BTE, called 3'TE-DR1, includes the 17-nt sequence conserved among all BTEs and folds into a similar secondary structure. It differs from the luteovirus BTE in three key ways: i) it has five rather than three stem-loops radiating from the central hub, ii) an adjacent upstream A-rich sequence (ARS), enhances the activity of 3'TEDR1 by recruiting poly(A)-binding protein (Iwakawa et al. 2012), and iii) no base-pairing to the 5' UTR is required (Iwakawa et al. 2012; Mizumoto et al. 2003). The $3^{\prime}$ end of the $3^{\prime}$ UTR of RNA1 contains other stem loop (SL) structures: SLDE, SLF, and intervening sequence $\mathrm{SeqB}$, which are essential for negative-strand RNA synthesis. 3'TE-DR1 is dispensable for RCNMV RNA1 negative-strand synthesis (Iwakawa et al. 2007).

There are two examples of trans-acting viral RNAs that control RCNMV gene expression. The first is a 34-nt RNA2 trans activator (TA) that is essential for the transcription of CPsgRNA from RNA1 (Sit et al. 1998), RNA2 replication (Basnayake et al. 2006; Tatsuta et al. 2005), and virion assembly (Basnayake et al. 2006). This sequence in RNA2 forms a stem-loop and the eight-base loop base pairs to a region in RNA1 two bases upstream of the start site of CPsgRNA (Sit et al. 1998). It is proposed that this base pairing sometimes blocks the replicase as it synthesizes (-) strand of RNA1, creating a truncated negative strand that serves as template for synthesis of (+) sense CPsgRNA (Sit et al. 1998). The TA of RNA2 is also essential for virion assembly as it acts as origin of assembly sequence and its interaction with RNA1 facilitates coencapsidation of RNA1 and RNA2 in the virion (Basnayake et al. 2009). Although this is a trans-acting RNA, it is not a noncoding RNA because the TA resides in the MP coding region. 
RNA1 of RCNMV generates a 431-nt ncsgRNA called SR1f, which comprises the $3^{\prime}$ UTR (Iwakawa et al. 2008). SR1f resembles BYDV sgRNA2 as follows: i) it is highly abundant, ii) it contains the BTE and the downstream frameshift element near its $5^{\prime}$ end and the replication origin at its $3^{\prime}$ end, iii) it inhibits translation of viral genomic RNA in trans, in vitro, and in vivo, iv) it inhibits translation of capped, polyadenylated, nonviral RNA in vitro and in vivo, $v$ ) it is not required for the virus to infect protoplasts or plants, although genomic RNA levels are lower when unable to produce SR1F (Iwakawa et al. 2008), and vi) the BTE in SR1f binds the eIF4G subunit of eIF4F with high affinity (Kraft et al. 2013). SR1f differs from BYDV sgRNA2 in that it i) is packaged in virions, ii) is half as long, iii) encodes no ORF, iv) contains the ARS upstream of the BTE, and v) is fully occupied with sequence required for translation or RNA synthesis, i.e., it lacks the tracts of 'mystery' sequence of unknown function in the luteovirus $3^{\prime}$ UTR.

A remarkable feature of SR1f is the mechanism by which it arises. SR1f is generated in the absence of RNA replication by a host exonuclease that degrades RNA1 in the $5^{\prime}$ to $3^{\prime}$ direction until the nuclease is blocked $431 \mathrm{nt}$ from the $3^{\prime}$ end by a $58-\mathrm{nt}$ structure called Seq1f58 (Fig. 3) (Iwakawa et al. 2008). This mechanism of SR1f generation may be identical to that of the ncsgRNA of flaviviruses (sfRNA), which is generated by exonuclease XRN1 (Pijlman et al. 2008). For both RCNMV and the flaviviruses, the RNA structure (xrRNA) that blocks the exonuclease is highly specialized. Mutations that disrupted secondary structure, removed the blockage and, thus, the sfRNA/SR1f RNA production, but compensating mutations predicted to restore the xrRNA structure did not restore the sfRNA or SRf1 RNA accumulation (Chapman et al. 2014b; Iwakawa et al. 2008). Thus, specific structures that are not easily predicted are required to block the exonuclease. X-ray crystallography revealed that the xrRNA of Murray valley encephalitis virus RNA forms a looped pseudoknot that binds tightly to XRN1 and is pulled tightly like a noose as the nuclease attempts to proceed in the $3^{\prime}$ direction
(Chapman et al. 2014b) Somewhat different structures achieve the same results in other flaviviruses (Chapman et al. 2014a; Clarke et al. 2015). In fact, xrRNA binds XRN1 so tightly and is so abundant that it sequesters the nuclease, preventing it from performing its normal duties in host mRNA turnover (Moon et al. 2012).

sfRNA has been shown to act as a sponge to sequester many host and viral proteins (Roby et al. 2014), including those needed to mount an interferon response (Bidet et al. 2014; Manokaran et al. 2015; Schuessler et al. 2012). West Nile virus sfRNA also inhibits the RNA interference (RNAi) response (Schnettler et al. 2012). sfRNA is not required for virus replication in cells or in mice but it greatly increases pathogenesis (Manokaran et al. 2015). While plants don't have an interferon response, it is an intriguing possibility that RCNMV SR1f RNA could affect the RNAi-based antiviral defense system, given the importance of RNAi in plant innate immunity.

RCNMV RNA lacks a 5' cap, so it may be particularly vulnerable to exonucleolytic degradation. Sequestration of the exonuclease (most likely XRN4 in plants) by SR1f may thus protect full-length viral RNAs from degradation. Finally, sfRNA may function like sgRNA2 of BYDV (Shen and Miller 2004; Shen et al. 2006) and, possibly, SR1f RNA of RCNMV (Iwakawa et al. 2012), by regulating viral translation in trans, as Fan et al. (2011) provided evidence that sfRNA modulates both translation and negative-strand synthesis of Japanese encephalitis virus in trans.

In addition to the SR1f RNA of RCNMV, ncsgRNAs corresponding to the 3' UTR have been identified in the following tombusvirids (Fig. 1): the machlomovirus Maize chlorotic mottle virus (Scheets 2000), the tombusvirus Cucumber necrosis virus (Johnston and Rochon 1995), and the betanecrovirus Tobacco necrosis virus-D (Jiwan et al. 2011). Similar to RCNMV RNA1 and unlike luteoviruses, these viruses have shorter (around 300 to $400 \mathrm{nt}$ ) 3' UTRs, which have the CITE near the $5^{\prime}$ end and the replication origin at the extreme $3^{\prime}$ end

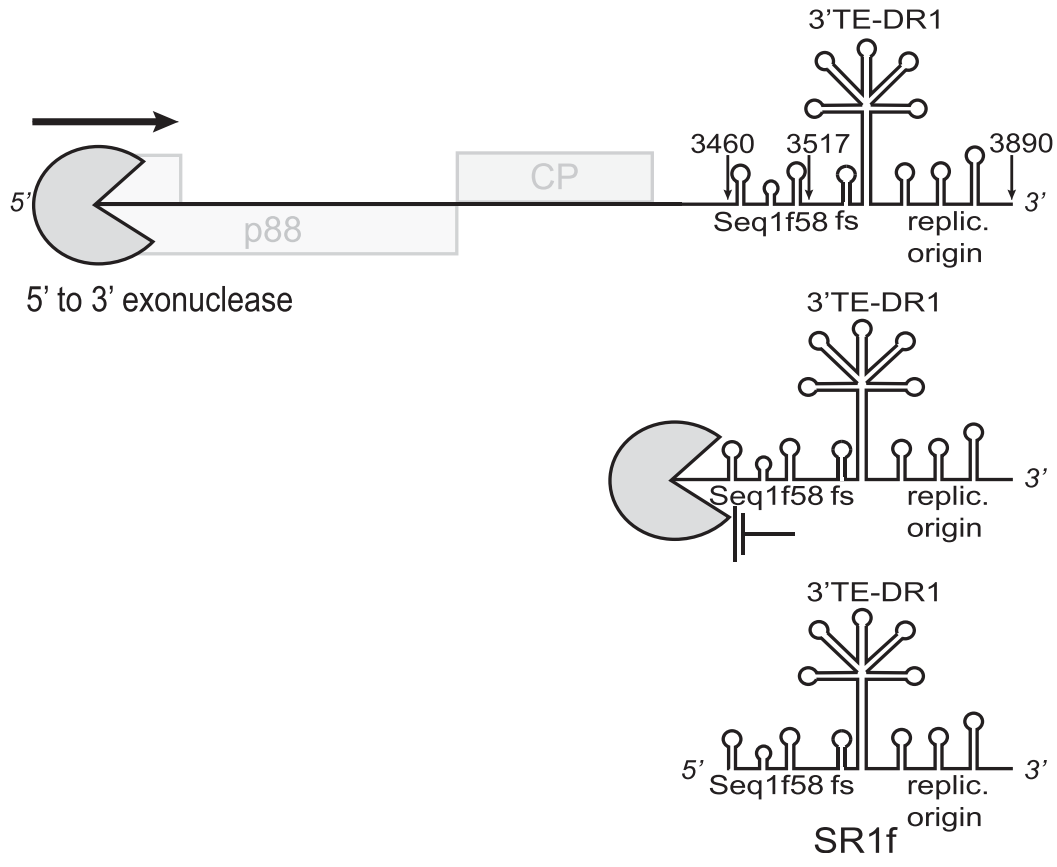

Fig. 3. Generation of the small noncoding RNA SR1f, spanning the 3' untranslated region of Red clover necrotic mosaic virus (RCNMV) RNA1 (not to scale). Host exonuclease degrades RNA1 from the 5' end until it is blocked by the Seq1f58 structure, leaving the stable product SR1f, comprising the 3' end of the genome, bases 3,460 to 3,890 (Iwakawa et al. 2008). Stem-loops shown in SR1f RNA, indicate i) Seq1f58, which is necessary and sufficient to block the exonuclease, ii) the downstream stem-loop required for frameshifting (fs), iii) the 3' Barley yellow dwarf virus-like translation element (3'TE-DR1), and iv) the $3^{\prime}$-proximal stem-loops required for initiation of replication (replic. origin). Not shown: it is possible that CPsgRNA generated from RCNMV RNA1 may also be a substrate for the exonuclease to generate SR1f RNA. 
(Fig. 1). Whether these ncsgRNAs are required for virus infection and their role in the virus life cycle is unknown.

It is possible that these ncsgRNAs as well as sgRNA3 of BYDV are generated by the same host exonuclease mechanism that produces SR1f RNA of RCNMV. The larger sgRNAs of BYDV, RCNMV, and other tombusvirids require RNA synthesis to accumulate and have sequences at their $5^{\prime}$ termini that resemble the $5^{\prime}$ end of the genome, presumably origins of $(+)$ strand synthesis on the negative strand (Jiwan and White 2011; Jiwan et al. 2011; Koev et al. 1999; Newburn and White 2015; Sit et al. 1998). In contrast, the small ncsgRNAs often lack any recognizable promoter-like sequences near their $5^{\prime}$ ends (Johnston and Rochon 1995; Koev and Miller 2000; Scheets 2000), supporting a different mechanism of production, such as via host exonuclease. In addition to genomic RNA, sgRNA1 and especially the abundant sgRNA2 of BYDV could also serve as substrates from which sgRNA3 is generated. This would explain why a point mutation, which completely prevents synthesis of sgRNA2, also reduces accumulation of sgRNA3 (Fig. 2A) (Shen et al. 2006). It is quite possible that other tombusvirids produce such ncsgRNAs but that they have been obscured by degradation products in low-resolution Northern blots or were simply ignored because they are too small to serve as subgenomic mRNAs and too large to be small RNAs involved in host RNAmediated defense.

\section{Cauliflower mosaic virus RNA jams the RNAi machinery.}

A DNA plant virus produces ncRNAs that appear to inhibit the RNAi system, as mentioned above for the flaviviruses and proposed as a possibility for RCNMV SR1f RNA. Cauliflower mosaic virus (CaMV) is a pararetrovirus with a nicked, doublestranded circular DNA genome (Hohn and Rothnie 2013). In infected cells, massive amounts of 20- to 25-nt viral small RNAs (vsRNAs) mapping to both strands of the highly structured 600-nt leader sequence of the viral 35S RNA accumulate
(Blevins et al. 2011). This leader is transcribed as a separate 600-nt 8S RNA, whose function has been a mystery since its discovery in 1982 (Guilley et al. 1982). The 8S RNA is likely generated by initiation of transcription at the $35 \mathrm{~S}$ promoter and termination by polymerase run-off at a nick in the negative strand DNA located 600 nt downstream (Blevins et al. 2011).

CaMV levels do not increase in plants with the RNAi defense system eliminated; $r d r 1 / 2 / 6$ triple knockouts and $d c l 1 / 2 / 3 / 4$ quadruple knockouts had no effect on CaMV levels (Blevins et al. 2011). Thus, the RNA-based defense system is ineffective against CaMV. The 8S transcript is highly structured and may resemble viroid RNA (Hemmings-Mieszczak et al. 1997), which is recognized and replicated by host DNA-dependent RNA polymerase II (pol II) (Rackwitz et al. 1981). Thus, Blevins et al. (2011) speculate that the negative strand of the 8S RNA is also generated by pol II. They propose that the massive amounts of vsRNAs generated from the $8 \mathrm{~S}$ dsRNA serve as decoys to overwhelm the RNAi silencing complex (RISC) and prevent it from generating enough vsRNAs that target the rest of the genome. Indeed, immunoprecipitation of AGO1, the major component of the RISC, revealed that almost all of the coimmunoprecipitating RNA associated with AGO1 mapped to the 8S RNA derived from the 600 -nt leader sequence and almost none to the rest of the genome (Blevins et al. 2011). Thus, the antiviral defense system is targeted almost entirely to the abundant, noncoding 8S transcript. Owing to its high degree of secondary structure, few vsRNAs gain access to their complementary targets in 8S RNA (or the same sequences in the leader of the $35 \mathrm{~S}$ RNA), rendering the virus unscathed by the RNAi machinery (Hohn 2015). This leaves the genomic 35S RNA and the other viral mRNA (19S RNA) free to express viral genes and allows the 35S RNA to serve as template for viral genome replication.

The role of the 8S RNA in jamming host RNAi-mediated defense was further supported by transferring the $8 \mathrm{~S}$ DNA sequence into a completely different kind of virus, Cabbage leaf

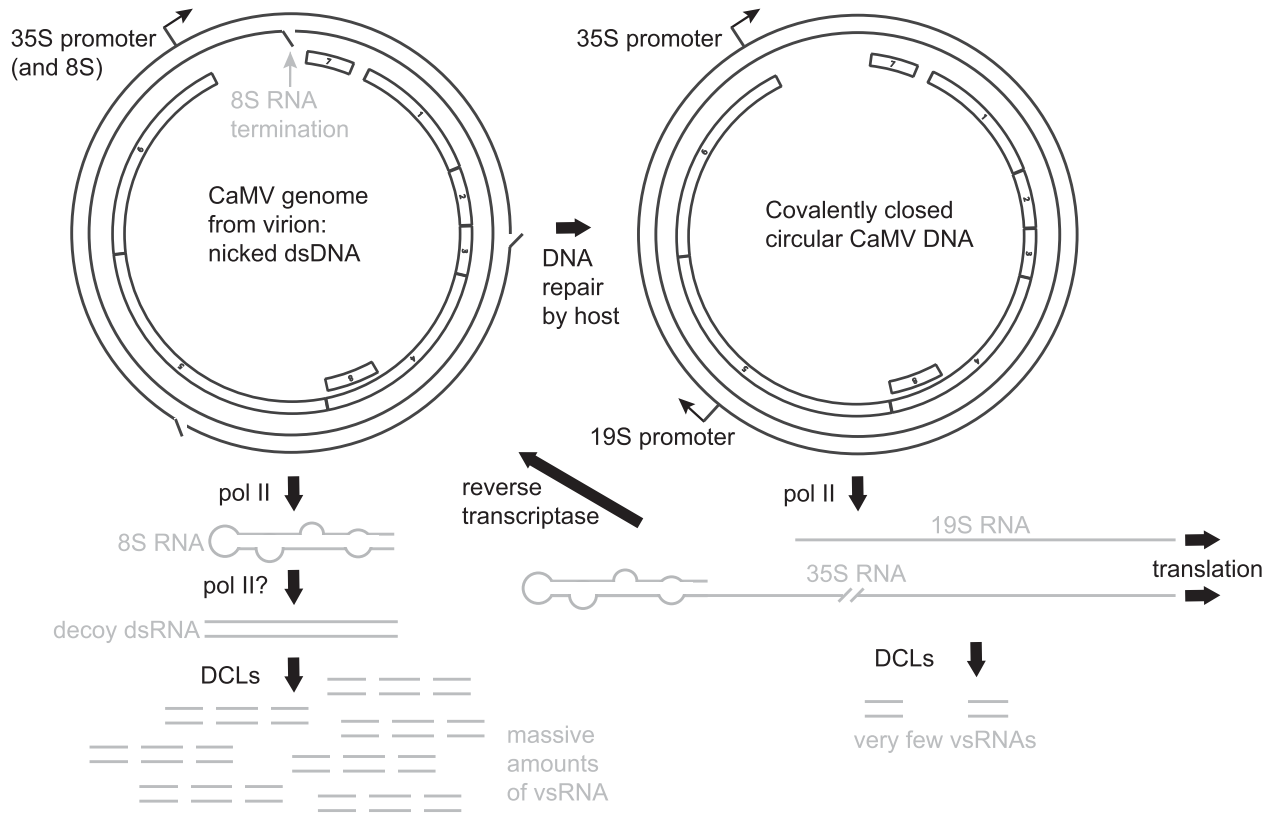

Fig. 4. Transcription of massive levels of noncoding RNA from the 5' leader of Cauliflower mosaic virus (CaMV) 35S RNA (modified from Blevins et al. [2011]). Circular, double-stranded DNA genome is shown with open reading frames inside. Left, transcription of the nicked, double stranded circular genome of CaMV from the $35 \mathrm{~S}$ promoter results in early termination at the first nick, giving large quantities of $8 \mathrm{~S}$ RNA comprising the highly structured untranslated leader sequence. This RNA may be copied into double stranded (ds)RNA) via pol II, owing to its resemblence to viroid RNA. Any of the DCL proteins then cleave this RNA into 20- to 25-nt viral small RNAs (vsRNAs) that flood the RNA-induced silencing complex, preventing the defense system from making effective amounts of small interfering RNA to target the larger viral RNAs. At right, host enzymes repair the nicks, creating the covalently closed dsDNA template from which full-length 35S and 19S RNAs are transcribed. Both serve as mRNAs, and 35S RNA also serves as the genomic replication intermediate that is reverse transcribed to make more nicked, genomic dsDNA. 
curl virus $(\mathrm{CaLCuV})$, a geminivirus. $\mathrm{CaLCuV}$ expressing this leader sequence replicated to higher levels than virus expressing only vector RNA, and large amounts of vsRNAs corresponding to the $8 \mathrm{~S}$ RNA sequence in the modified $\mathrm{CaLCuV}$ accumulated (Blevins et al. 2011). To summarize, the authors propose a model in which the nicked, circular form of CaMV DNA is transcribed to produce the 8S RNA (noncoding leader sequence of $35 \mathrm{~S}$ RNA), which is then copied by pol II into dsRNA that is processed by the Dicer-like (DCL) proteins into massive amounts of vsRNAs that overload the RISC machinery (AGO1 protein), preventing the RNAi response from inhibiting virus infection (Fig. 4). For CaMV genome replication, host DNA repair enzymes remove the nicks in virion DNA, preventing termination of transcription that generates the 8S RNA, while allowing transcription of the normal $35 \mathrm{~S}$ and $19 \mathrm{~S}$ viral RNAs needed for virus gene expression and replication (Fig. 4). Given the high structure and abundance of the ncsgRNAs of tombusvirid and luteovirus 3' UTRs (discussed above), we speculate that they, too, have the potential to overwhelm the RNAi-based defense system in a similar fashion.

\section{Viroid-derived small RNAs can target specific host mRNAs to reduce their expression and enhance pathogenesis.}

Viroids consist of 250 to $400 \mathrm{nt}$ circular ncRNAs with a high degree of self-complementarity, causing them to form rod-shaped, partially double-stranded structures. As with viral infections, small (21 to $24 \mathrm{nt}$ ) viroid RNAs (vd-sRNAs) accumulate in plants infected with viroids, owing to processing by DCL and AGO proteins in the RNAi-based antiviral defense system (Minoia et al. 2015; Navarro et al. 2012; Tsushima et al. 2015). Emerging evidence indicates that some vd-sRNAs (Flores et al. 2015; AdkarPurushothama et al. 2015) as well as a satellite RNA of Cucumber mosaic virus (Shimura et al. 2011; Smith et al. 2011) can function like host miRNA to specifically target and degrade host mRNAs.

Strains of Peach latent mosaic viroid (PLMVd) that cause a 'peach calico' (PC) or albino symptom contain a 12-nt hairpin insertion. In infected tissue that has the albino phenotype, a 21-nt vd-sRNA, which includes this 12-nt insertion, accumulates. This vd-sRNA can base-pair to mRNA encoding the chloroplast heat-shock protein 90 (cHSP90). This results in cleavage of the mRNA as predicted for miRNA-mediated cleavage (Navarro et al. 2012). The vd-sRNA accumulates and cHSP90 mRNA is cleaved only in the albino tissue. This degradation of cHSP90 mRNA may favor viroid accumulation, as somewhat higher levels of PLMVd RNA accumulate in the albino tissue relative to the green tissue in which cHSP90 mRNA is not cleaved (Flores et al. 2015). Thus, the PC strains of PLMVd may be turning the RNAimediated defense system of the host to their own advantage.

A more clear-cut example of this exploitation of the RNAi system by a viroid was discovered recently for Potato spindle tuber viroid (PSTVd) (Adkar-Purushothama et al. 2015). As few as two base differences in the pathogenicity-determining domain of PSTVd RNA can greatly affect symptom severity and replication efficiency (Tsushima et al. 2015). AdkarPurushothama et al. (2015) showed that this region is processed by the host DCL proteins and RISC to produce a vd-sRNA that modulates host gene expression to the advantage of the viroid. One of the vd-sRNAs in tomato plants infected with a severe strain of PSTVd (PSTVd-I) has partial complementarity to mRNA encoding the callose synthase 11-like protein (CalS11like), whereas the homologous vd-sRNA from a mild strain of PSTVd (PSTVd-M) has much weaker complementarity to CalS11-like mRNA. Expression of CalS11-like mRNA was reduced more in PSTVd-I-infected than in PSTVd-M-infected plants. Expression of the severe 21-nt vd-sRNA sequence in the context of a miRNA, in the absence of PSTVd infection, reduced expression of a green fluorescent protein reporter gene containing the predicted target sequence in its $3^{\prime}$ UTR. These data plus results of additional experiments support the hypothesis that the vd-sRNA generated by the host antiviral RNAi machinery functionally mimics a miRNA that targets host callose synthesis mRNAs, thereby reducing mRNA levels and, presumably, callose levels. This, in turn, enhances viroid accumulation and movement in the plant because callose synthesis at the plasmodesmata is a host defense mechanism known to reduce virus movement from cell to cell through the plasmodesmata ( $\mathrm{Li}$ et al. 2012). Indeed, it had been shown previously that the sequence of the pathogenicity domains that generate these key vd-sRNAs controls efficiency of viroid cell-to-cell movement (Zhong et al. 2008). Thus in both PLMVd and PSTVd infections, ncRNA generated from the viroid genome simply incorporates itself into the RNAi system of the host plant to negatively regulate expression of a presumed host defense gene in order to facilitate more efficient infection.

It remains a mystery why, to our knowledge and that of Flores et al. (2015), only viroids and satellite RNAs are known to produce small RNAs that knock down expression of host genes, while plant viral genomes are not known to (naturally) produce small RNAs (vsRNAs) that directly target host mRNAs, reducing their expression. This is despite observations that i) vsRNAs accumulate to substantial levels in most plant viral infections, ii) some of these vsRNAs are complementary to host mRNAs (Qi et al. 2009), and iii) artificial insertion of host sequences into viral genomes allows many plant viruses to serve as efficient tools for virus-induced gene silencing of host genes (Mysore and Senthil-Kumar 2015).

\section{Conclusion: Viral RNAs are often sponges of proteins.}

All viral RNAs, coding and noncoding, compete with host RNAs and with each other for interactions with host and viral proteins, complexes, and organelles in a way that results in productive infection. Thus viral RNAs can be considered sponges of host proteins and larger complexes (Charley and Wilusz 2014). In most cases, such as positive-strand RNA virus genomic RNAs and subgenomic mRNAs, the RNAs efficiently bind translation factors, effectively sponging them away from host mRNAs, and they bind other host proteins and membranes (Nagy et al. 2012; Pathak et al. 2011), sponging them away from their normal cellular processes (Clarke et al. 2015). This can hinder the ability of the host to mount a defense response (Bidet et al. 2014; Charley and Wilusz 2014). Only recently have plant virologists become aware that ncRNAs of plant viruses, often unnoticed despite their high abundance, may play such a role in plant virus infection. Here, we have provided examples of such RNAs that can reduce the amount of translation machinery, RNAi machinery, or defense gene mRNA available to the cell, thus favoring infection.

A common feature of ncRNAs is that they are not essential for virus infection. Knockout mutations that prevent expression of viral ncRNAs do not prevent virus replication (Fig. 2A) (Clarke et al. 2015; Iwakawa et al. 2008; Miller et al. 2015). Similarly, viroids with sequence variations that prevent vdsRNAs from mimicking defense gene-targeted miRNAs are still able to replicate in plants (Adkar-Purushothama et al. 2015; Flores et al. 2015). However, viruses and viroids that generate functional ncRNAs are known (or likely) to have a competitive advantage for optimal accumulation by attenuating the defense response of the host. This lack of essentiality and lack of coding regions may explain why so few ncRNAs have been identified or discussed much in publications that reveal them. For example, an sfRNA of a flavivirus was first reported in 1997 (Urosevic et al. 1997), but its significance was not realized until 2008 (Pijlman et al. 2008; Roby et al. 2014). For 
these reasons, we may have missed some other published ncRNAs of plant viruses (for that we apologize). We predict that many more trans-acting ncRNAs exist for many plant viruses and still await discovery.

\section{ACKNOWLEDGMENTS}

The authors thank K. A. White for imparting some of his vast knowledge of viral RNAs and $\mathrm{S}$. Whitham for valuable discussions on RNAi and virusinduced gene silencing. This work was funded by National Institute of Health grant no. R01GM067104 to W. A. Miller . This journal paper of the Iowa Agriculture and Home Economics Experiment Station, Ames, IA Project No. 3708 was supported in part by Hatch Act and State of Iowa funds.

\section{LITERATURE CITED}

Adkar-Purushothama, C. R., Brosseau, C., Giguère, T., Sano, T., Moffett, P. and Perreault, J.-P. 2015. Small RNA derived from the virulence modulating region of the Potato spindle tuber viroid silences callose synthase genes of tomato plants. Plant Cell 27:2178-2194.

Barry, J. K., and Miller, W. A. 2002. A -1 ribosomal frameshift element that requires base pairing across four kilobases suggests a mechanism of regulating ribosome and replicase traffic on a viral RNA. Proc. Natl. Acad. Sci. U.S.A. 99:11133-11138.

Basnayake, V. R., Sit, T. L., and Lommel, S. A. 2006. The genomic RNA packaging scheme of Red clover necrotic mosaic virus. Virology 345 : 532-539.

Basnayake, V. R., Sit, T. L., and Lommel, S. A. 2009. The Red clover necrotic mosaic virus origin of assembly is delimited to the RNA-2 trans-activator. Virology 384:169-178.

Bidet, K., Dadlani, D., and Garcia-Blanco, M. A. 2014. G3BP1, G3BP2 and CAPRIN1 are required for translation of interferon stimulated mRNAs and are targeted by a dengue virus non-coding RNA. PLoS Pathog. 10: e1004242.

Blevins, T., Rajeswaran, R., Aregger, M., Borah, B. K., Schepetilnikov, M., Baerlocher, L., Farinelli, L., Meins, F., Jr., Hohn, T., and Pooggin, M. M. 2011. Massive production of small RNAs from a non-coding region of Cauliflower mosaic virus in plant defense and viral counter-defense. Nucleic Acids Res. 39:5003-5014.

Brown, C. M., Dinesh-Kumar, S. P., and Miller, W. A. 1996. Local and distant sequences are required for efficient readthrough of the barley yellow dwarf virus PAV coat protein gene stop codon. J. Virol. 70 5884-5892.

Chalhoub, B. A., Kelly, L., Robaglia, C., and Lapierre, H. D. 1994. Sequence variability in the genome-3'-terminal region of BYDV for 10 geographically distinct PAV-like isolates of barley yellow dwarf virus: Analysis of the ORF6 variation. Arch. Virol. 139:403-416.

Chapman, E. G., Costantino, D. A., Rabe, J. L., Moon, S. L., Wilusz, J., Nix, J. C., and Kieft, J. S. 2014b. The structural basis of pathogenic subgenomic flavivirus RNA (sfRNA) production. Science 344:307-310.

Chapman, E. G., Moon, S. L., Wilusz, J., and Kieft, J. S. 2014a. RNA structures that resist degradation by Xrn1 produce a pathogenic Dengue virus RNA. eLife 3:e01892.

Charley, P. A., and Wilusz, J. 2014. Sponging of cellular proteins by viral RNAs. Curr. Opin. Virol. 9:14-18.

Clarke, B. D., Roby, J. A., Slonchak, A., and Khromykh, A. A. 2015. Functional non-coding RNAs derived from the flavivirus 3' untranslated region. Virus Res. 206:53-61.

Clough, S. J., and Bent, A. F. 1998. Floral dip: A simplified method for Agrobacterium-mediated transformation of Arabidopsis thaliana. Plant J. 16:735-743.

Dinesh-Kumar, S. P., and Miller, W. A. 1993. Control of start codon choice on a plant viral RNA encoding overlapping genes. Plant Cell 5:679-692.

Dolja, V. V., and Koonin, E. V. 2011. Common origins and host-dependent diversity of plant and animal viromes. Curr. Opin. Virol. 1:322-331.

Domier, L. L. 2012. Family Luteoviridae. Pages 1045-1053 in: Virus Taxonomy: Ninth Report of the International Committee on the Taxonomy of Viruses. A. M. Q. King, M. J. Adams, E. B. Carstens, and E. J. Lefkowitz, eds. Elsevier Academic Press, Amsterdam.

Domier, L. L., McCoppin, N. K., Larsen, R. C., and D’Arcy, C. J. 2002. Nucleotide sequence shows that Bean leafroll virus has a Luteovirus-like genome organization. J. Gen. Virol. 83:1791-1798.

Fan, Q., Treder, K., and Miller, W. A. 2012. Untranslated regions of diverse plant viral RNAs vary greatly in translation enhancement efficiency. BMC Biotechnol. 12:22.
Fan, Y.-H., Nadar, M., Chen, C.-C., Weng, C.-C., Lin, Y.-T., and Chang, R.-Y. 2011. Small noncoding RNA modulates japanese encephalitis virus replication and translation in trans. Virol. J. 8:492.

Flores, R., Minoia, S., Carbonell, A., Gisel, A., Delgado, S., LópezCarrasco, A., Navarro, B., and Di Serio, F. 2015. Viroids, the simplest RNA replicons: How they manipulate their hosts for being propagated and how their hosts react for containing the infection. Virus Res. 209: 136-145.

Gago-Zachert, S. 2016. Viroids, infectious long non-coding RNAs with autonomous replication. Virus Res. 212:12-24.

Guilley, H., Dudley, R. K., Jonard, G., Balàzs, E., and Richards, K. E. 1982. Transcription of cauliflower mosaic virus DNA: Detection of promoter sequences, and characterization of transcripts. Cell 30:763-773.

Guo, L., Allen, E. M., and Miller, W. A. 2001. Base-pairing between untranslated regions facilitates translation of uncapped, nonpolyadenylated viral RNA. Mol. Cell 7:1103-1109.

Guo, Y. E., and Steitz, J. A. 2014. Virus meets host microRNA: The destroyer, the booster, the hijacker. Mol. Cell. Biol. 34:3780-3787.

Hemmings-Mieszczak, M., Steger, G., and Hohn, T. 1997. Alternative structures of the cauliflower mosaic virus 35 S RNA leader: Implications for viral expression and replication. J. Mol. Biol. 267:1075-1088.

Hohn, T. 2015. RNA based viral silencing suppression in plant pararetroviruses. Front. Plant Sci. 6:398.

Hohn, T., and Rothnie, H. 2013. Plant pararetroviruses: Replication and expression. Curr. Opin. Virol. 3:621-628.

Hollingsworth, M. J., Kim, J.-K., and Stollar, N. E. 1998. Heelprinting analysis of in vivo ribosome pause sites. Pages 153-165 in: Protein Synthesis: Methods and Protocols. R. Martin, ed. Humana Press, Totowa, NJ, U.S.A.

Iwakawa, H. O., Kaido, M., Mise, K., and Okuno, T. 2007. cis-Acting core RNA elements required for negative-strand RNA synthesis and capindependent translation are separated in the $3^{\prime}$-untranslated region of Red clover necrotic mosaic virus RNA1. Virology 369:168-181.

Iwakawa, H. O., Mizumoto, H., Nagano, H., Imoto, Y., Takigawa, K. Sarawaneeyaruk, S., Kaido, M., Mise, K., and Okuno, T. 2008. A vira noncoding RNA generated by cis-element-mediated protection against $5^{\prime} \rightarrow 3^{\prime}$ RNA decay represses both cap-independent and cap-dependent translation. J. Virol. 82:10162-10174.

Iwakawa, H. O., Tajima, Y., Taniguchi, T., Kaido, M., Mise, K., Tomari, Y. Taniguchi, H., and Okuno, T. 2012. Poly(A)-binding protein facilitates translation of an uncapped/nonpolyadenylated viral RNA by binding to the $3^{\prime}$ untranslated region. J. Virol. 86:7836-7849.

Jiwan, S. D., and White, K. A. 2011. Subgenomic mRNA transcription in Tombusviridae. RNA Biol. 8:287-294.

Jiwan, S. D., Wu, B., and White, K. A. 2011. Subgenomic mRNA transcription in tobacco necrosis virus. Virology 418:1-11.

Johnston, J. C., and Rochon, D. M. 1995. Deletion analysis of the promoter for the cucumber necrosis virus 0.9-kb subgenomic RNA. Virology 214: 100-109.

Kelly, L., Gerlach, W. L., and Waterhouse, P. M. 1994. Characterisation of the subgenomic RNAs of an Australian isolate of barley yellow dwarf luteovirus. Virology 202:565-573.

Koev, G., Liu, S., Beckett, R., and Miller, W. A. 2002. The $3^{\prime}$-terminal structure required for replication of barley yellow dwarf virus RNA contains an embedded 3' end. Virology 292:114-126.

Koev, G., and Miller, W. A. 2000. A positive-strand RNA virus with three very different subgenomic RNA promoters. J. Virol. 74:5988-5996.

Koev, G., Mohan, B. R., and Miller, W. A. 1999. Primary and secondary structural elements required for synthesis of barley yellow dwarf virus subgenomic RNA1. J. Virol. 73:2876-2885.

Kraft, J. J., Treder, K., Peterson, M. S., and Miller, W. A. 2013. Cationdependent folding of $3^{\prime}$ cap-independent translation elements facilitates interaction of a 17-nucleotide conserved sequence with eIF4G. Nucleic Acids Res. 41:3398-3413.

Li, W., Zhao, Y., Liu, C., Yao, G., Wu, S., Hou, C., Zhang, M., and Wang, D. 2012. Callose deposition at plasmodesmata is a critical factor in restricting the cell-to-cell movement of Soybean mosaic virus. Plant Cell Rep. 31:905-916.

Liu, Y., Zhai, H., Zhao, K., Wu, B., and Wang, X. 2012. Two suppressors of RNA silencing encoded by cereal-infecting members of the family Luteoviridae. J. Gen. Virol. 93:1825-1830.

Lommel, S. A., Weston-Fina, M., Xiong, Z., and Lomonossoff, G. P. 1988 The nucleotide sequence and gene organization of red clover necrotic mosaic virus RNA-2. Nucleic Acids Res. 16:8587-8602.

Manokaran, G., Finol, E., Wang, C., Gunaratne, J., Bahl, J., Ong, E. Z., Tan, H. C., Sessions, O. M., Ward, A. M., Gubler, D. J., Harris, E., GarciaBlanco, M. A., and Ooi, E. E. 2015. Dengue subgenomic RNA binds TRIM25 to inhibit interferon expression for epidemiological fitness. Science 350:217-221. 
Miller, W. A., Jackson, J., and Feng, Y. 2015. Cis- and trans-regulation of luteovirus gene expression by the $3^{\prime}$ end of the viral genome. Virus Res. 206:37-45.

Miller, W. A., and Koev, G. 2000. Synthesis of subgenomic RNAs by positive-strand RNA viruses. Virology 273:1-8.

Miller, W. A., Liu, S., and Beckett, R. 2002. Barley yellow dwarf virus: Luteoviridae or Tombusviridae? Mol. Plant Pathol. 3:177-183.

Minoia, S., Navarro, B., Delgado, S., Di Serio, F., and Flores, R. 2015. Viroid RNA turnover: Characterization of the subgenomic RNAs of potato spindle tuber viroid accumulating in infected tissues provides insights into decay pathways operating in vivo. Nucleic Acids Res. 43:2313-2325.

Mizumoto, H., Tatsuta, M., Kaido, M., Mise, K., and Okuno, T. 2003. Capindependent translational enhancement by the $3^{\prime}$ untranslated region of Red clover necrotic mosaic virus RNA1. J. Virol. 77:12113-12121.

Moon, S. L., Anderson, J. R., Kumagai, Y., Wilusz, C. J., Akira, S., Khromykh, A. A., and Wilusz, J. 2012. A noncoding RNA produced by arthropod-borne flaviviruses inhibits the cellular exoribonuclease XRN1 and alters host mRNA stability. RNA 18:2029-2040.

Mysore, K. S., and Senthil-Kumar, M., eds. 2015. Plant Gene Silencing: Methods and Protocols. Humana Press, New York.

Nagy, P. D., Barajas, D., and Pogany, J. 2012. Host factors with regulatory roles in tombusvirus replication. Curr. Opin. Virol. 2:691-698.

Navarro, B., Gisel, A., Rodio, M. E., Delgado, S., Flores, R., and Di Serio, F. 2012. Small RNAs containing the pathogenic determinant of a chloroplast-replicating viroid guide the degradation of a host mRNA as predicted by RNA silencing. Plant J. 70:991-1003.

Newburn, L. R., and White, K. A. 2015. Cis-acting RNA elements in positive-strand RNA plant virus genomes. Virology 479-480:434-443.

Okuno, T., and Hiruki, C. 2013. Molecular biology and epidemiology of dianthoviruses. Adv. Virus Res. 87:37-74.

Palukaitis, P. 2016. Satellite RNAs and satellite viruses. Mol. Plant-Microbe Interact 29:Xxx-Xxx.

Pathak, K. B., Pogany, J., and Nagy, P. D. 2011. Non-template functions of the viral RNA in plant RNA virus replication. Curr. Opin. Virol. 1:332-338.

Pijlman, G. P., Funk, A., Kondratieva, N., Leung, J., Torres, S., van der Aa, L., Liu, W. J., Palmenberg, A. C., Shi, P. Y., Hall, R. A., and Khromykh, A. A. 2008. A highly structured, nuclease-resistant, noncoding RNA produced by flaviviruses is required for pathogenicity. Cell Host Microbe 4:579-591.

Qi, X., Bao, F. S., and Xie, Z. 2009. Small RNA deep sequencing reveals role for Arabidopsis thaliana RNA-dependent RNA polymerases in viral siRNA biogenesis. PLoS One 4:e4971.

Rackwitz, H. R., Rohde, W., and Sänger, H. L. 1981. DNA-dependent RNA polymerase II of plant origin transcribes viroid RNA into full-length copies. Nature 291:297-301.

Rakotondrafara, A. M., Polacek, C., Harris, E., and Miller, W. A. 2006. Oscillating kissing stem-loop interactions mediate $5^{\prime}$ scanningdependent translation by a viral $3^{\prime}$-cap-independent translation element. RNA 12:1893-1906.

Roby, J. A., Pijlman, G. P., Wilusz, J., and Khromykh, A. A. 2014. Noncoding subgenomic flavivirus RNA: Multiple functions in West Nile virus pathogenesis and modulation of host responses. Viruses 6:404-427.

Rochon, D., Lommel, S. A., Martelli, G. P., Rubino, L., and Russo, M. 2012. Tombusviridae. Pages 1111-1138 in: Virus Taxonomy: Ninth report of the International Committee on Taxonomy of Viruses. A. M. Q. King, M. J. Adams, E. B. Carstens, and E. J. Lefkowitz, eds. Elsevier, San Diego.

Salem, N. M., Miller, W. A., Rowhani, A., Golino, D. A., and Moyne, A.-L., Falk, B. W. 2008. Rose spring dwarf-associated virus has RNA structural and gene-expression features like those of Barley yellow dwarf virus. Virology 375:354-360.

Scheets, K. 2000. Maize chlorotic mottle machlomovirus expresses its coat protein from a $1.47-\mathrm{kb}$ subgenomic RNA and makes a 0.34-kb subgenomic RNA. Virology 267:90-101.

Schnettler, E., Sterken, M. G., Leung, J. Y., Metz, S. W., Geertsema, C., Goldbach, R. W., Vlak, J. M., Kohl, A., Khromykh, A. A., and Pijlman, G. P. 2012. Noncoding flavivirus RNA displays RNA interference suppressor activity in insect and Mammalian cells. J. Virol. 86: 13486-13500.

Schuessler, A., Funk, A., Lazear, H. M., Cooper, D. A., Torres, S., Daffis, S., Jha, B. K., Kumagai, Y., Takeuchi, O., Hertzog, P., Silverman, R., Akira, S., Barton, D. J., Diamond, M. S., and Khromykh, A. A. 2012. West Nile virus noncoding subgenomic RNA contributes to viral evasion of the type I interferon-mediated antiviral response. J. Virol. 86:5708-5718.
Shen, R., and Miller, W. A. 2004. Subgenomic RNA as a riboregulator: Negative regulation of RNA replication by Barley yellow dwarf virus subgenomic RNA 2. Virology 327:196-205.

Shen, R., Rakotondrafara, A. M., and Miller, W. A. 2006. trans regulation of cap-independent translation by a viral subgenomic RNA. J. Virol. 80: 10045-10054.

Shen, W. J., and Forde, B. G. 1989. Efficient transformation of Agrobacterium spp. by high voltage electroporation. Nucleic Acids Res. 17:8385.

Shimura, H., and Masuta, C. 2016. Plant subviral RNAs as a long noncoding RNA (lncRNA): Analogy with animal lncRNAs in host-virus interactions. Virus Res. 212:25-29.

Shimura, H., Pantaleo, V., Ishihara, T., Myojo, N., Inaba, J., Sueda, K., Burgyán, J., and Masuta, C. 2011. A viral satellite RNA induces yellow symptoms on tobacco by targeting a gene involved in chlorophyll biosynthesis using the RNA silencing machinery. PLoS Pathog. 7: e 1002021 .

Simon, A. E., and Miller, W. A. 2013. 3' cap-independent translation enhancers of plant viruses. Annu. Rev. Microbiol. 67:21-42.

Sit, T. L., Vaewhongs, A. A., and Lommel, S. A. 1998. RNA-mediated trans-activation of transcription from a viral RNA. Science 281: 829-832.

Smirnova, E., Firth, A. E., Miller, W. A., Scheidecker, D., Brault, V., Reinbold, C., Rakotondrafara, A. M., Chung, B. Y., and Ziegler-Graff, V. 2015. Discovery of a small non-AUG-initiated ORF in Poleroviruses and Luteoviruses that is required for long-distance movement. PLoS Pathog. 11:e1004868.

Smith, N. A., Eamens, A. L., and Wang, M. B. 2011. Viral small interfering RNAs target host genes to mediate disease symptoms in plants. PLoS Pathog. 7:e1002022.

Tajima, Y., Iwakawa, H. O., Kaido, M., Mise, K., and Okuno, T. 2011. A long-distance RNA-RNA interaction plays an important role in programmed -1 ribosomal frameshifting in the translation of $\mathrm{p} 88$ replicase protein of Red clover necrotic mosaic virus. Virology 417:169-178.

Tatsuta, M., Mizumoto, H., Kaido, M., Mise, K., and Okuno, T. 2005. The Red clover necrotic mosaic virus RNA2 trans-activator is also a cisacting RNA2 replication element. J. Virol. 79:978-986.

Treder, K., Kneller, E. L., Allen, E. M., Wang, Z., Browning, K. S., and Miller, W. A. 2008. The $3^{\prime}$ cap-independent translation element of Barley yellow dwarf virus binds eIF4F via the eIF4G subunit to initiate translation. RNA 14:134-147.

Tsushima, D., Adkar-Purushothama, C., Taneda, A., and Sano, T. 2015. Changes in relative expression levels of viroid-specific small RNAs and microRNAs in tomato plants infected with severe and mild symptominducing isolates of Potato spindle tuber viroid. J. Gen. Plant Pathol. 81: 49-62.

Urosevic, N., van Maanen, M., Mansfield, J. P., Mackenzie, J. S., and Shellam, G. R. 1997. Molecular characterization of virus-specific RNA produced in the brains of flavivirus-susceptible and -resistant mice after challenge with Murray Valley encephalitis virus. J. Gen. Virol. 78:23-29.

Wang, S., Browning, K. S., and Miller, W. A. 1997. A viral sequence in the $3^{\prime}$-untranslated region mimics a $5^{\prime}$ cap in facilitating translation of uncapped mRNA. EMBO J. 16:4107-4116.

Wang, S., Guo, L., Allen, E., and Miller, W. A. 1999. A potential mechanism for selective control of cap-independent translation by a viral RNA sequence in cis and in trans. RNA 5:728-738.

Xiong, Z., Kim, K. H., Giesman-Cookmeyer, D., and Lommel, S. A. 1993. The roles of the red clover necrotic mosaic virus capsid and cell-to-cell movement proteins in systemic infection. Virology 192:27-32.

Xiong, Z., and Lommel, S. A. 1989. The complete nucleotide sequence and genome organization of red clover necrotic mosaic virus RNA-1. Virology 171:543-554.

Yamagishi, N., Terauchi, H., Kanematsu, S., and Hidaka, S. 2003. Characterization of the small subgenomic RNA of Soybean dwarf virus Arch. Virol. 148:1827-1834.

Zavriev, S. K., Hickey, C. M., and Lommel, S. A. 1996. Mapping of the red clover necrotic mosaic virus subgenomic RNA. Virology 216:407-410.

Zhong, X., Archual, A. J., Amin, A. A., and Ding, B. 2008. A genomic map of viroid RNA motifs critical for replication and systemic trafficking. Plant Cell 20:35-47.

Zuo, J., Niu, Q. W., and Chua, N. H. 2000. An estrogen receptorbased transactivator XVE mediates highly inducible gene expression in transgenic plants. Plant J. 24:265-273. 